\title{
A Duality Theorem for the Automorphism Group of a Covariant System
}

\author{
JOHN ERNEST \\ University of California at Santa Barbara
}

Received November 5, 1969

\begin{abstract}
In this paper we examine the covariant representation theory of a covariant system $(A, G)$ introduced by Doplicher, Kastler and Robinson. ( $A$ is a $C^{*}$-algebra and $G$ is a locally compact group of automorphisms of $A$.) We define the concept of left tensor product of two covariant representations. Loosely stated, our duality theorem says that $G$ is canonically isomorphic to the set of bounded operator valued maps on the set of covariant representations of the covariant system $(A, G)$ which preserve direct sums, unitary equivalence and left tensor products. We further show that the enveloping von Neumann algebra $\mathscr{A}(A, G)$ of the covariant system $(A, G)$ admits a (not necessarily injective) comultiplication $d$ such that $(\mathscr{A}(A, G), d)$ is a Hopf von Neumann algebra. The intrinsic group of this Hopf von Neumann algebra is canonically isomorphic and (strong operator topology) homeomorphic to $G$.
\end{abstract}

\section{§ 1. Introduction}

Generally speaking, the mathematical purpose of any representation theory is to study an abstract and perhaps intractable object by examining the collection of structure preserving maps (morphisms) into some simpler, or at least more concrete object. In the case of the unitary representation theory of locally compact groups, the term "concrete" is more appropriate than the term "simpler" as every locally compact group is isomorphic and homeomorphic to a group of unitary operators acting on a Hilbert space, in the strong operator topology (cf. Lemma 2.2 of [2]). A similar phenomena of course shows up in the case of the *-representation theory of a $C^{*}$-algebra.

If the examination of the representation theory is to serve as an effective tool for obtaining information about the structure of the original abstract object, we must have a representation theory which completely determines the structure of that object. One of the most basic questions one can ask of any representation theory is: Are there enough representations? Initially we would like to know if there are enough representations to distinguish points. Once we have satisfied ourselves as to this minimal requirement of any representation theory, 
we would like to know if the collection of representations, together with whatever natural structure this collection possesses, is sufficiently rich to completely determine the structure of the abstract object. Any precise answer to such a question will put into focus the properties of the representation theory which are particularly relevant for determining the structure of the abstract object, as well as suggest how information about the representation theory could be translated into information about the object being represented. In the case of locally compact Abelian groups, for example, the beautiful Pontrjagen duality theorem tells us that the morphisms of the group into the circle group, together with their multiplication and a natural topology, determines the group completely. Because of the special elegant form of this result $(\xi \cong G)$ any theorem indicating how to construct a represented object from its representations is now referred to as a duality theorem. Thus we have the Tannaka duality theorem for compact groups, [5], the Tatsuuma duality theorem for locally compact groups [6] and the Takesaki duality theorem for separable $C^{*}$-algebras [4] (a clean sweep for the Kyoto school).

In this paper we examine the duality question for the covariant systems considered by S. Doplicher, D. Kastler and D. Robinson in [1]. A covariant system is a pair $(A, G)$ consisting of a $C^{*}$-algebra $A$ and a locally compact group $G$, of automorphisms of $A$ (cf. Definition 2.1 of [3]). We show that the automorphism group $G$ may be reconstructed from the set of covariant representations of the system. To do this we require additional structure on the set of covariant representations. Thus we are led to introduce the concept of left tensor products of covariant representations. Loosely stated, our theorem says that $G$ is canonically isomorphic to the set of bounded operator valued maps on the set of covariant representations of the covariant system, which preserve direct sums, unitary equivalence and left tensor products.

In the previous paper [3], we characterized the enveloping von Neumann algebra of a covariant system $(A, G)$ as the set of options on the covariant representations, i.e., the bounded maps on the covariant representations which preserve direct sums and unitary equivalence (cf. Definition 3.2 of [3]). Since the covariant system $(A, G)$ is isomorphically embedded in $\mathscr{A}(A, G)$ (cf. Theorem 3.5 of [3]), the duality problem may be formulated as the question of identifying the automorphism group $G$, as a group of unitary elements in the algebra $\mathscr{A}(A, G)$. In this paper we show that $\mathscr{A}(A, G)$ admits a natural (not necessarily injective) comultiplication $d$ such that the pair $(\mathscr{A}(A, G), d)$ is a Hopf von Neumann algebra (cf. [2]). (The comultiplication $d$ is a normal *-homomorphism of $\mathscr{A}(A, G)$ into $\mathscr{A}(A, G) \otimes \mathscr{A}(A, G)$.) The duality theorem may then be formulated as saying that $G$ may be identified as 
the intrinsic group of the Hopf von Neumann algebra. By the intrinsic group of $(\mathscr{A}(A, G), d)$, we mean the group of invertible elements $T$ in $\mathscr{A}(A, G)$ for which $d(T)=T \otimes T$.

In Section 2 we define the various notions of tensor products which may be applied to covariant representations. In Section 3 we show that $\mathscr{A}(A, G)$ is a Hopf von Neumann algebra. We also show that the covariant enveloping algebra $\mathscr{A}_{c}(G)$, of $G$ (cf. $\S 4$ of [3]) is a Hopf von Neumann algebra. We also prove in this section, that in the embedding of the covariant system $(A, G)$ into the enveloping algebra $\mathscr{A}(A, G), A$ and $G$ have at most the identity element of $\mathscr{A}(A, G)$, in common. In Section 4 we consider the left $\pi_{0}$-regular covariant representation $(\pi, L)$ of $(A, G)$ (cf. Definition 3.6 of [3]), where $\pi_{0}$ is any non-zero $*$-representation of $A$. We show that the von Neumann algebra $\mathscr{A}(\pi, L)$ generated by the range of $(\pi, L)$ is a Hopf von Neumann algebra and its intrinsic group is precisely the set of operators $\left\{L_{x}: x \in G\right\}$, which is isomorphic and (strong operator topology) homeomorphic to $G$. In Section 5 we show that the intrinsic group of $\mathscr{A}(A, G)$ is precisely $G$, as embedded canonically in $\mathscr{A}(A, G)$. In Section 6 we state and prove this result in a form completely analogous to the classical Tannaka duality theorem for compact groups [5].

A portion of this work was done while the author was a visitor at the Mathematisches Institut der Universität, at Göttingen, Germany, during the summer of 1969 and he wishes to express his gratefulness for the hospitality of the Institute. He also thanks the National Science Foundation, USA for its financial support.

\section{§ 2. Left and Right Tensor Products of Covariant Representations}

Let $\mathscr{H}_{0}$ denote a fixed Hilbert space of sufficiently large dimension such that every proper cyclic covariant repesentation of $(A, G)$ is unitary equivalent to one whose representation space is a closed subspace of $\mathscr{H}_{0}$ (cf. Proposition 2.7 and Definition 3.1 of [3]). Let $\mathscr{R}(A, G)$ denote the set of all proper covariant representations of $(A, G)$ which act on some closed subspace of $\mathscr{H}_{0}$.

In order to identify the automorphism group elements in the enveloping algebra $\mathscr{A}(A, G)$ we need some additional structure in the set $\mathscr{R}(A, G)$. Thus we wish to introduce some concept of "tensor product" among covariant representations. The basic idea here is that we form the usual tensor product of the unitary group representations and then amplify the *-representation of the $C^{*}$-algebra $A$ in order to obtain a new covariant representation. 
Proposition 2.1. Let $(\pi, U)$ denote a covariant representation of the covariant system $(A, G)$, and let $V$ denote any strongly continuous unitary representation of $G$. Let I denote the identity operator on $\mathscr{H}(V)$. Then $(\pi \otimes I, U \otimes V)$ and $(I \otimes \pi, V \otimes U)$ are covariant representations of $(A, G)$.

Proof. The representation $x \rightarrow \pi(x) \otimes I$ is a $*$-representation of $A$ on $\mathscr{H}(U) \otimes \mathscr{H}(V)$ known as the amplification of $\pi$. In fact $\pi \otimes I$ is unitary equivalent to $\alpha \pi$ where $\alpha=\operatorname{dim} \mathscr{H}(V)$. Then for any $x \in A, s \in G$ we have

$$
\begin{aligned}
(\pi \otimes I)(s(x)) & =\pi(s(x)) \otimes I \\
& =U(s) \pi(x) U(s)^{*} \otimes V(s) I V(s)^{*} \\
& =[U(s) \otimes V(s)] \cdot[\pi(x) \otimes I] \cdot[U(s) \otimes V(s)]^{*} \\
& =[(U \otimes V)(s)] \cdot[(\pi \otimes I)(x)] \cdot[(U \otimes V)(s)]^{*} .
\end{aligned}
$$

Remark 2.2. Let $\mathscr{R}_{2}(A, G)$ denote the set of all unitary representations $U$ of $G$ such that, there exists a $*$-representation $\pi$ of $A$ for which $(\pi, U) \in \mathscr{R}(A, G)$. Thus $\mathscr{R}_{2}(A, G)$ is a subset of $G^{c}$, the set of all strongly continuous unitary representations acting on a closed subspace of $\mathscr{H}_{0}$. Clearly $\mathscr{R}_{2}(A, G)$ is closed under the operations of direct sum and unitary equivalence in the sense that if $U_{1}$ and $U_{2}$ are contained in $\mathscr{R}_{2}(A, G)$ and $U_{1} \oplus U_{2} \in G^{c}$, then $U_{1} \oplus U_{2} \in \mathscr{R}_{2}(A, G)$. Similarly if $u$ is a linear isometry such that $U_{2}=u U_{1} u^{*}$ and $U_{1} \in \mathscr{R}_{2}(A, G)$ and $U_{2} \in G^{c}$, then $U_{2} \in \mathscr{R}_{2}(A, G)$. The previous proposition then states that $\mathscr{R}_{2}(A, G)$ is the analogue of an ideal in $G^{c}$, relative to the representation theoretic operations in $G^{c}$.

Definition 2.3. Let $\left(\pi_{1}, U_{1}\right)$ and $\left(\pi_{2}, U_{2}\right)$ denote two covariant representations of the covariant system $(A, G)$.

The left tensor product, denoted $\left(\pi_{1}, U_{1}\right) Q\left(\pi_{2}, U_{2}\right)$, is defined to be

$$
\left(\pi_{1} \otimes I, U_{1} \otimes U_{2}\right) \text {. }
$$

The right tensor product, denoted $\left(\pi_{1}, U_{1}\right) \varnothing\left(\pi_{2}, U_{2}\right)$ is defined to be

$$
\left(I \otimes \pi_{2}, U_{1} \otimes U_{2}\right) \text {. }
$$

Remark 2.4. Clearly these binary operations are not commutative. In fact

$$
\left(\pi_{1}, U_{1}\right) Q\left(\pi_{2}, U_{2}\right)=\left(\pi_{2}, U_{2}\right) \varnothing\left(\pi_{1}, U_{1}\right) .
$$

If this asymmetry is too bothersome, one can also define the symmetric tensor product by

$$
\left(\pi_{1}, U_{1}\right) \otimes\left(\pi_{2}, U_{2}\right)=\left[\left(\pi_{1}, U_{1}\right) Q\left(\pi_{2}, U_{2}\right)\right] \oplus\left[\left(\pi_{1}, U_{1}\right) \varnothing\left(\pi_{2}, U_{2}\right)\right] .
$$


For the purpose of duality we shall need only a minimal amount of extra structure for the covariant representations and it would be sufficient to define the left tensor square of a covariant representation $(\pi, U)$ by

$$
(\pi, U)^{Q}=(\pi, U) Q(\pi, U) .
$$

\section{§ 3. Hopf von Neumann Algebras}

In an earlier paper [2], we defined a Hopf von Neumann algebra $\mathscr{A}$ as a von Neumann algebra admitting an associative comultiplication. In that paper we required the comultiplication to be an isomorphism of $\mathscr{A}$ into $\mathscr{A} \otimes \mathscr{A}$. In this paper we are forced to relax this condition, by requiring only that the comultiplication be a normal $*$-homomorphism of $\mathscr{A}$ into $\mathscr{A} \otimes \mathscr{A}$. However this new definition, which we now give formally, is in fact more consistent with the notion of Hopf algebra as employed by algebraists.

Definition 3.1. A Hopf von Neumann algebra is a von Neumann algebra $\mathscr{A}$ that admits an associative comultiplication, i.e., a normal *-homomorphism $d$ of $\mathscr{A}$ into $\mathscr{A} \otimes \mathscr{A}$ such that

$$
(d \otimes i) \circ d=(i \otimes d) \circ d
$$

where $i$ denotes the identity map on $\mathscr{A}$.

A Hopf von Neumann algebra is said to be cocommutative if $d=\Phi d$, where $\Phi$ is the canonical isomorphism of $\mathscr{A} \otimes \mathscr{A}$ onto $\mathscr{A} \otimes \mathscr{A}$ defined by $\Phi(S \otimes T)=T \otimes S$, for all $S, T$ in $\mathscr{A}$.

We remark that in general, unlike the situation in [2], the Hopf von Neumann algebras considered in this paper will not be cocommutative. This is a consequence of the asymmetry present in defining tensor product structure among covariant representations. Indeed the reader can clearly see that the comultiplication defined in the next theorem is not cocommutative.

Theorem 3.2. The enveloping von Neumann algebra $\mathscr{A}(A, G)$ of a covariant system, is a Hopf von Neumann algebra. In fact, $\mathscr{A}(A, G)$ admits a unique associative comultiplication

$$
d: \mathscr{A}(A, G) \rightarrow \mathscr{A}(A, G) \otimes \mathscr{A}(A, G)
$$

such that

$$
d(x)=x \otimes I \quad \text { for all } x \text { in } A
$$

and

$$
d(s)=s \otimes s \quad \text { for all } s \text { in } G .
$$

Here I denotes the identity element of $\mathscr{A}(A, G)$.

6 Commun. math Phys, Vol. 17 
Proof. Note that this comultiplication is uniquely determined by the conditions stated since $d$ is a $\sigma$-strongly continuous homomorphism and $A \cup G$ generates $\mathscr{A}(A, G)$ (cf. Theorem 3.5 of [3]).

Consider the universal representation $(v, V)$ of $(A, G)$, obtained by taking the direct sum of all the proper covariant representations in $\mathscr{R}(A, G)$. Then by theorem 3.7 of [3], this has a unique extension to a normal $*$-homomorphism of $\mathscr{A}(A, G)$ onto $\mathscr{A}(v, V)$. This is in fact a *-isomorphism and hence represents $\mathscr{A}(A, G)$ faithfully as an algebra of operators acting on a very large Hilbert space (cf. the proof of Theorem 3.3 of [3]). Let $\varphi$ denote this isomorphism of $\mathscr{A}(A, G)$ onto $\mathscr{A}(v, V)$. Then the left tensor square $(v, V)^{Q}$ of $(v, V)$ also has a unique extension to a normal *-representation $d^{\prime}$ of $\mathscr{A}(A, \mathrm{G})$. Further the range of $d^{\prime}$ is generated by the set $\{v(x) \otimes I: x \in A\} \cup\{V(s) \otimes V(s): s \in G\}$. Hence the range of $d^{\prime}$ is contained in $\mathscr{A}(v, V) \otimes \mathscr{A}(v, V)$, which is mapped isomorphically onto $\mathscr{A}(A, G) \otimes \mathscr{A}(A, G)$ by $\varphi^{-1} \otimes \varphi^{-1}$. Thus $d=\left(\varphi^{-1} \otimes \varphi^{-1}\right) \circ d^{\prime}$ is a normal $*$-homomorphism of $\mathscr{A}(A, G)$ into $\mathscr{A}(A, G) \otimes \mathscr{A}(A, G)$.

It remains to verify that $d$ is associative. But since $d$ is a normal *-homomorphism, it is sufficient to verify the equation

$$
(d \otimes i) \circ d=(i \otimes d) \circ d
$$

on the generating set $A \cup G$ of $\mathscr{A}(A, G)$. But for $s \in G$, both sides yield $s \otimes s \otimes s$. For $x \in A$, both sides yield $x \otimes I \otimes I$, where $I$ is the identity of $\mathscr{A}(A, G)$.

This theorem leads us to the observation that $A$ and $G$ are essentially disjoint in $\mathscr{A}(A, G)$.

Proposition 3.3. Consider the covariant system $(A, G)$ to be embedded in its enveloping von Neumann algebra $\mathscr{A}(A, G)$. Then:

(1) If $A$ contains an identity element, then $A \cap G$ consists of the single element, which is at the same time the identity element of $A$, of $G$, and of $\mathscr{A}(A, G)$.

(2) If $A$ does not contain an identity, then $A \cap G$ is empty. Further the approximate identity of $A$ converges strongly to the identity of $\mathscr{A}(A, G)$.

Proof. It is easy to verify that if $I$ is the identity element of $A$, then its corresponding option on $\mathscr{R}(A, G)$ is the identity of $\mathscr{A}(A, G)$ and is exactly the option corresponding to the identity element of $\mathrm{G}$.

If $x$ is an element of $A$ and $x=s$ for some $s \in G$ we have $d(x)=x \otimes I$ $=s \otimes I$ while $d(x)=s \otimes s$. Thus $s \otimes I=s \otimes s$ and hence $x=s=I$. Hence the identity is the only element which $G$ and $A$ have in common. Thus if $A$ does not have an identity we have $A \cap G=\emptyset$, the empty set. 
To see that the approximate identity converges strongly to the identity element of $\mathscr{A}(A, G)$, consider the isomorphism of $\mathscr{A}(A, G)$ onto $\mathscr{A}(v, V)$ where $(v, V)$ is the universal covariant representation of $\mathscr{A}(A, G)$. Then $v$ is a proper representation and hence the projection onto the essential space of $v$ is exactly the identity operator which is the identity of $\mathscr{A}(v, V)$. Thus the result follows from Lemma 2.4 of [3].

Definition 3.4. An element $S$ of a Hopf von Neumann algebra $(\mathscr{A}, d)$ is called an intrinsic element of $\mathscr{A}$ if $S$ is invertible and $d(S)=S \otimes S$.

Clearly the intrinsic elements of any Hopf von Neumann algebra form a group, which we call the intrinsic group of the algebra. In our previous work (cf. Proposition 3.6 of [2]) we showed that the intrinsic elements are in fact unitary. However in that argument we assumed the comultiplication $d$ was an isometry. Thus we sketch a modified proof which is applicable to this more general situation where $d$ need only be a normal $*$-homomorphism.

Proposition 3.5. The elements of the intrinsic group of any Hopf von Neumann algebra are unitary.

Proof. First note that if $S$ is any intrinsic element of a Hopf von Neumann algebra $(\mathscr{A}, d)$, then $\|S\| \geqq\|d(S)\|=\|S \otimes S\|=\|S\|^{2}$. Hence $\|S\| \leqq 1$ for any intrinsic element.

Let $T$ be an intrinsic element of $(\mathscr{A}, d)$. Then one may verify that $T T^{*}$ and its inverse are intrinsic elements. By the previous result, $\left\|T^{*} T\right\| \leqq 1$ and $\left\|\left(T^{*} T\right)^{-1}\right\| \leqq 1$, from which one concludes that in fact $\left\|T^{*} T\right\|=1$ and $\left\|\left(T^{*} T\right)^{-1}\right\|=1$. Since $T^{*} T$ is a positive operator, the spectral theorem implies $T^{*} T=I$. Similarly $T T^{*}=I$.

Remark 3.6. Note that the covariant enveloping algebra $\mathscr{A}_{c}(G)$ of $G$, defined as the von Neumann subalgebra of $\mathscr{A}(A, G)$ generated by $G$, is in fact a Hopf von Neumann subalgebra of $\mathscr{A}(A, G)$ (cf. $\S 4$ of [3]). Indeed, let $d^{\prime}$ denote the restriction of the comultiplication $d$ to the subalgebra $\mathscr{A}_{c}(G)$. Then the range of $d^{\prime}$ is contained in $\mathscr{A}(A, G) \otimes \mathscr{A}(A, G)$. However since $G$ generates $\mathscr{A}_{c}(G)$ and $d$ is a $\sigma$-strong continuous homomorphism, the range of $d^{\prime}$ is generated by $\{d(s)=s \otimes s: s \in G\}$. Hence the range of $d^{\prime}$ is contained in $\mathscr{A}_{c}(G) \otimes \mathscr{A}_{c}(G)$ and $\left(\mathscr{A}_{c}(G), d^{\prime}\right)$ is a Hopf von Neumann algebra.

Let $\left(\mathscr{A}(G), d^{\prime \prime}\right)$ denote the Hopf von Neumann enveloping algebra of $G$ (cf. [2]). Let $\varphi$ denote the canonical normal $*$-homomorphism of $\mathscr{A}(G)$ onto $\mathscr{A}_{c}(G)$ (cf. $\S 4$ of [3]). Then $d^{\prime} \circ \varphi=(\varphi \otimes \varphi) \circ d^{\prime \prime}$ since they are both normal $*$-homomorphisms which agree on the generating set $G$. Hence the canonical map $\varphi$ of $\mathscr{A}(G)$ onto $\mathscr{A}_{c}(G)$ described in $\S 4$ of [3], is in fact a Hopf von Neumann algebra morphism. 


\section{$\S 4$. The Left $\pi_{0}$-regular Covariant Representation}

We first recall a definition from [3].

Definition 4.1. Let $(A, G)$ be a covariant system and let $\pi_{0}$ denote any non-zero *-representation of $A$. Let $\mu$ denote left invariant Haar measure on $G$ and let $L^{2}\left(G, \mathscr{H}\left(\pi_{0}\right)\right)$ denote the space of all $\mu$-square integrable measurable functions of $G$ into $\mathscr{H}\left(\pi_{0}\right)$. We define the left $\pi_{0}$-regular covariant representation $(\pi, L)$ in the space $L^{2}\left(G, \mathscr{H}\left(\pi_{0}\right)\right)$ by

$$
(L(s) \xi)(t)=\xi\left(s^{-1} t\right) \quad \text { for all } s, t \text { in } G
$$

and

$$
(\pi(x) \xi)(t)=\pi_{0}\left(t^{-1} x\right) \xi(t) \quad \text { for all } x \text { in } A, t \text { in } G .
$$

Theorem 4.2. Let $\pi_{0}$ be a proper *-representation of $A$, where $(A, G)$ is a covariant system. Let $(\pi, L)$ denote the left $\pi_{0}$-regular covariant representation of $(A, G)$.

Let $U$ be a strongly continuous unitary representation of $G$, on $\mathscr{H}(U)$. By Proposition $2.1(\pi \otimes I, L \otimes U)$ is a covariant representation of $(A, G)$ on $L^{2}\left(G, \mathscr{H}\left(\pi_{0}\right)\right) \otimes \mathscr{H}(U)$. Then $(\pi \otimes I, L \otimes U)$ is quasi-equivalent to $(\pi, L)$. More precisely, if $\alpha=\operatorname{dim} \mathscr{H}(U)$, then $(\pi \otimes I, L \otimes U)$ is unitary equivalent to a direct sum of $\alpha$ copies of $(\pi, L)$.

Indeed let $\left\{\varphi_{\lambda}\right\}$ denote an orthonormal basis for $\mathscr{H}$. Then the map $\varphi$, defined by

$$
\varphi: \xi \otimes \eta \rightarrow\left\{\left(\eta, U(\cdot) \varphi_{\lambda}\right) \xi(\cdot)\right\}
$$

of

$$
L^{2}\left(G, \mathscr{H}\left(\pi_{0}\right)\right) \otimes \mathscr{H}(U) \quad \text { onto } \quad \sum_{\lambda \in A} \oplus L^{2}(G, \mathscr{H}(\pi))
$$

is a linear isometry such that

$$
\varphi(\pi \otimes I) \varphi^{-1}=\sum_{\lambda \in \Lambda} \oplus \pi
$$

and

$$
\varphi(L \otimes U) \varphi^{-1}=\sum_{\lambda \in A} \oplus L .
$$

Proof. Clearly $\varphi$ is well-defined and linear. We verify that $\varphi$ is an isometry. Since $U$ is a unitary representation, for each fixed $t$ in $G$, $\left\{U(t) \varphi_{\lambda}\right\}$ is an orthonormal basis for $\mathscr{H}(U)$. Hence

$$
\begin{aligned}
\sum_{\lambda \in A} \|(\eta, U(t) & \left.\varphi_{\lambda}\right) \xi(t) \|^{2} \\
& =\|\xi(t)\|^{2} \sum_{\lambda \in \Lambda}\left|\left(\eta, U(t) \varphi_{\lambda}\right)\right|^{2} \\
& =\|\xi(t)\|^{2}\|\eta\|^{2} .
\end{aligned}
$$


Hence

$$
\begin{aligned}
\|\left\{\left(\eta, U(\cdot) \varphi_{\lambda}\right)\right. & \xi(\cdot)\} \|^{2} \\
& =\sum_{\lambda}\left\|\left(\eta, U(\cdot) \varphi_{\lambda}\right) \xi(\cdot)\right\|^{2} \\
& =\sum_{\lambda} \int_{G}\left\|\left(\eta, U(t) \varphi_{\lambda}\right) \xi(t)\right\|^{2} d \mu(t) \\
& =\int_{G}\left(\sum_{\lambda}\left\|\left(\eta, U(t) \varphi_{\lambda}\right) \xi(t)\right\|^{2}\right) d \mu(t) \\
& =\int_{G}\|\xi(t)\|^{2}\|\eta\|^{2} d \mu(t) \\
& =\|\xi\|^{2}\|\eta\|^{2} .
\end{aligned}
$$

Thus

$$
\| \varphi(\xi \otimes \eta\|=\| \xi\|\| \eta\|=\| \xi \otimes \eta \|
$$

We next verify that $\varphi$ is surjective. For a fixed $\lambda_{0}$, let $E_{\lambda_{0}}$ denote the projection of $\sum_{\lambda \in A} \oplus L^{2}\left(G, \mathscr{H}\left(\pi_{0}\right)\right)$ onto the $\lambda_{0}$-component. Consider the continuous complex valued function on $G, f(x)=\left(\varphi_{\lambda_{0}}, U(x) \varphi_{\lambda_{0}}\right)$. Since $f(e)=1$, there exists an open neighborhood $N$ of $e$ such that $f$ is bounded away from 0 on $N$. Suppose $t \in G$ and $\xi$ is an element of $L^{2}\left(G, \mathscr{H}\left(\pi_{0}\right)\right)$ such that $\xi(x)=0$ for all $x$ not in $x$ not in $t N$. Letting $f_{t}(x)=f\left(t^{-1} x\right)$ for all $x$ in $G$, we define $\xi^{\prime}$ in $L^{2}\left(G, \mathscr{H}\left(\pi_{0}\right)\right)$ by $\xi^{\prime}(x)=\frac{\xi(x)}{f_{t}(x)}$ if $x \in t N$ and $\xi^{\prime}(x)=0$ if $x \notin t N$. Then $\xi(x)=\left(U(t) \varphi_{\lambda_{0}}, U(x) \varphi_{\lambda_{0}}\right) \xi^{\prime}(x)$ for all $x$ in $G$. Thus we have established that if $\xi \in L^{2}\left(G, \mathscr{H}\left(\pi_{0}\right)\right)$ and if $\xi$ vanishes outside $t N$ for some $t$ in $G$, then $\xi$ is in the range of $E_{\lambda_{0}}{ }^{\circ} \varphi$.

Now let $g$ be a continuous function from $G$ into $\mathscr{H}\left(\pi_{0}\right)$, with compact support $K$. Then there exists $t_{1}, \ldots, t_{n}$ in $G$ such that $K \subset \bigcup_{i=1}^{n} t_{i} N$. Let $M_{1}=t_{1} N$ and let $M_{i}=t_{i} N-\left(\bigcup_{j=1}^{i-1} t_{j} N\right)$, for $i=2, \ldots, n$. Then the $M_{i}$ are mutually disjoint measurable sets such that $M_{i} \subset t_{i} N, i=1, \ldots, n$, and $K \subset \bigcup_{i=1}^{n} M_{i}$. Let $\chi_{M_{i}}$ denote the characteristic function of $M_{i}$, $i=1, \ldots, n$. Then $\chi_{M_{\imath}} g$ is in the range of $E_{\lambda_{0}} \circ \varphi$ by our previous result, and

$$
g=\sum_{i=1}^{n} \chi_{M_{i}} g
$$

Since $\varphi$ is linear, $g$ is in the range of $E_{\lambda_{0}} \circ \varphi$. Thus $E_{\lambda_{0}} \circ \varphi$ is a continuous map of a Hilbert space onto a dense subspace of $L^{2}\left(G, \mathscr{H}_{0}\right)$ and hence is surjective. Since $\lambda_{0}$ was arbitrary, $\varphi$ is surjective. 
We leave to the reader the task of verifying that $\varphi$ does map

$$
(\pi \otimes I, L \otimes U) \quad \text { into } \quad \sum_{\lambda \in A} \oplus(\pi, L) .
$$

Theorem 4.3. Let $\pi_{0}$ denote a proper *-representation of $A$, where $(A, G)$ is a covariant system. Let $(\pi, L)$ denote the left $\pi_{0}$-regular covariant representation of $(A, G)$ and, as usual, let $\mathscr{A}(\pi, L)$ denote the von Neumann algebra generated by the range of $(\pi, L)$.

Then $\mathscr{A}(\pi, L)$ admits a unique injective comultiplication $r$ such that $(\mathscr{A}(\pi, L), r)$ is a Hopf von Neumann algebra for which

$$
r(\pi(x))=\pi(x) \otimes I \quad \text { for all } x \text { in } A
$$

and

$$
r(L(s))=L(s) \otimes L(s) \quad \text { for all } s \text { in } G .
$$

Further the intrinsic group of $\mathscr{A}(\pi, L)$ is precisely the set

$$
\{L(s): s \in G\} .
$$

Indeed the map $s \rightarrow L(s)$ is a isomorphism and ( $\sigma$-strong) homeomorphism of $G$ onto the intrinsic group of $(\mathscr{A}(\pi, L), r)$.

Proof. Consider the left tensor square $(\pi, L)^{\natural}$ of $(\pi, L)$. By the previous theorem $(\pi, L)^{\otimes}=(\pi \otimes I, L \otimes L)$ is quasi-equivalent to $(\pi, L)$. Hence (cf. the remark following Proposition 2.7 in [3]) there exists an $*$-isomorphism $r$ of $\mathscr{A}(\pi, L)$ onto $\mathscr{A}\left((\pi, L)^{\otimes}\right)$ such that

$$
r(\pi(x))=\pi(x) \otimes I \quad \text { for all } x \text { in } A
$$

and

$$
r(L(s))=L(s) \otimes L(s) \quad \text { for all } s \text { in } G
$$

Note that $\mathscr{A}\left((\pi, L)^{\otimes}\right) \subset \mathscr{A}(\pi, L) \otimes \mathscr{A}(L) \subset \mathscr{A}(\pi, L) \otimes \mathscr{A}(\pi, L)$. Hence $r$ maps $\mathscr{A}(\pi, L)$ into $\mathscr{A}(\pi, L) \otimes \mathscr{A}(\pi, L)$. Also note that $r$ is uniquely determined by the given conditions since $r$ is a normal $*$-homomorphism and $\mathscr{A}(\pi, L)$ is generated by $\{\pi(x), L(s): x \in A, s \in G\}$. To see that the comultiplication $r$ is associative we must verify the formula $(r \otimes i) \circ r=(i \otimes r) \circ r$. For this purpose it is sufficient to verify the formula for the generating elements of $\mathscr{A}(\pi, L)$. However both sides of the formula, when applied to $\pi(x)$, for $x$ in $A$, yield $\pi(x) \otimes I \otimes I$. Similarly both sides, when applied to $L(s)$, for $s$ in $G$, yield $L(s) \otimes L(s) \otimes L(s)$.

Suppose now that $y$ is an intrinsic element of $\mathscr{A}(\pi, L)$. Then $r(y)=y \otimes y$. Further, as noted above, $r$ maps $\mathscr{A}(\pi, L)$ into $\mathscr{A}(\pi, L) \otimes \mathscr{A}(L)$. Hence $y \in \mathscr{A}(L)$, the von Neumann algebra generated by the range of $L$. Since $L$ is quasi-equivalent to the left-regular representation $L_{0}$ (cf. the proof of Theorem 3.5 of [3]) and since the left regular representation has the 
property that it is quasi-equivalent to its own tensor square (a special case of Theorem 4.2) we have that $L$ is quasi-equivalent to $L \otimes L$. Since $L \sim L_{0}$ we have that there is an isomorphism $\varphi$ of $\mathscr{A}(L)$ onto $\mathscr{A}\left(L_{0}\right)$ such that $\varphi(L(s))=L_{0}(s)$ for all $\sin G$. The restriction $r^{\prime}$ of $r$ to $\mathscr{A}(L) \subset \mathscr{A}(\pi, \mathrm{L})$ is thus a comultiplication for $\mathscr{A}(L)$ such that $r^{\prime}(L(s))=L(s) \otimes L(s)$ for all $s$ in $G$. Since $\{L(s): s \in G\}$ generates $\mathscr{A}(L)$, this condition uniquely determines the comultiplication $r^{\prime}$ of $\mathscr{A}(L)$. Indeed, if we identify $\mathscr{A}(L)$ and $\mathscr{A}\left(L_{0}\right)$ by the above isomorphism, $r^{\prime}$ is precisely the comultiplication considered in Proposition 3.4 of [2]. Thus if $y$ is an intrinsic element of $\mathscr{A}(\pi, L)$ we have seen it must be contained in $\mathscr{A}(L)$ and is thus an intrinsic element of $\left.(\mathscr{A} L), r^{\prime}\right)$. By the Tatsuuma duality theorem (Proposition 3.8 of [2]), $y$ is necessarily of the form $y=L(s)$ for some $s$ in $G$.

The fact that $s \rightarrow L(s)$ is then an isomorphism and $\sigma$-strong homeomorphism simply follows from the fact that $L$ is quasi-equivalent to the left-regular representation $L_{0}$, and these properties hold for $L_{0}$ (cf. Lemma 2.2 of [2]).

Remark 4.4. Since we can construct a left $\pi_{0}$-regular covariant representation $(\pi, L)$ for each proper $*$-representation of $A$, we in fact have a whole collection of Hopf von Neumann algebras to which the previous theorem applies. Of course quasi-equivalent left $\pi_{0}$-regular covariant representations give rise to isomorphic Hopf von Neumann algebras (cf. the remark following Proposition 2.7 of [3]). Following Theorem 3.9 of [3], call a central projection $E$ of $\mathscr{A}(A, G)$ a regular projection if the restriction of the induction $\mathscr{A}(A, G) \rightarrow \mathscr{A}(A, G)_{E}$, to the covariant system $(A, G)$, is a covariant representation quasi-equivalent to a left $\pi_{0}$-regular covariant representation of $(A, G)$, for some proper *representation $\pi_{0}$ of $A$. Then for every regular central projection $E$, the algebra $\mathscr{A}(A, G)_{E}$ is a Hopf von Neumann algebra and the induction

$$
\mathscr{A}(A, G) \rightarrow \mathscr{A}(A, G)_{E}
$$

is a Hopf von Neumann algebra morphism.

\section{§ 5. The Enveloping Algebra Version of the Duality Theorem}

In an earlier work (cf. Remark 3.3 of [2]) we defined the concept of inner tensor product of any two *-representations of a Hopf von Neumann algebra. The following lemma may be interpreted as stating that the extension of the left tensor product of two covariant representations, to the enveloping algebra $\mathscr{A}(A, G)$, is precisely the inner tensor product of the corresponding normal $*$-representations of $\mathscr{A}(A, G)$ (cf. Theorem 3.7 of [3]). 
Lemma 5.1. Let $\left(\pi_{1}, U_{1}\right)$ and $\left(\pi_{2}, U_{2}\right)$ denote proper covariant representations of the covariant system $(A, G)$ with extensions to normal *-representations $\pi_{1}^{\prime}$ and $\pi_{2}^{\prime}$ respectively of the enveloping Hopf von Neumann algebra $(\mathscr{A}(A, G), d)$. Then the canonical extension of $\left(\pi_{1}, U_{1}\right) Q\left(\pi_{2}, U_{2}\right)$ to $\mathscr{A}(A, G)$ is precisely

$$
\left(\pi_{1}^{\prime} \otimes \pi_{2}^{\prime}\right) \circ d .
$$

Proof. Since $\left(\pi_{1}^{\prime} \otimes \pi_{2}^{\prime}\right) \circ d$ is a normal $*$-representation of $\mathscr{A}(A, G)$, it is sufficient to verify that its restriction to $(A, G)$ is $\left(\pi_{1}, U_{1}\right) Q\left(\pi_{2}, U_{2}\right)$. However $\left(\pi_{1}^{\prime} \otimes \pi_{2}^{\prime}\right) \circ d(x)=\pi_{1}^{\prime} \otimes \pi_{2}^{\prime}(x \otimes I)=\pi_{1}(x) \otimes I$ for all $x$ in $A$. Similarly for $s$ in $G$,

$$
\left(\pi_{1}^{\prime} \otimes \pi_{2}^{\prime}\right) \circ d(s)=U_{1}(s) \otimes U_{2}(s) .
$$

Notation. Before we state and prove our next theorem, it will be helpful to establish some precise notation to be used throughout this section. Let $(v, V)$ denote the universal representation of $(A, G)$ (cf. the proof of Theorem 3.3 of [3]) and assume the enveloping von Neumann algebra is concretely represented as acting on the Hilbert space $\mathscr{H}(v, V)$. We identify $\mathscr{A}(A, G)$ and $\mathscr{A}(v, V)$ and the canonical extension of $(v, V)$ to $\mathscr{A}(A, G)$ is therefore the identity map $i$.

Let $\pi_{0}$ be a proper $*$-representation of $A$ and let $(\pi, L)$ denote the left $\pi_{0}$-regular covariant representation of $(A, G)$. By the Theorem 4.3 $(\mathscr{A}(\pi, L), r)$ is a Hopf von Neumann algebra whose intrinsic group is precisely the set $\{L(s): s \in G\}$. Then (cf. Theorem 3.9 of [3]) there exists a central projection $E$ of $\mathscr{A}(A, G)$ such that the restriction $\left(\pi^{\prime}, L^{\prime}\right)$ of the induction

$$
e: \mathscr{A}(A, G) \rightarrow \mathscr{A}(A, G)_{E}
$$

to $(A, G)$, is a covariant representation quasi-equivalent to $(\pi, L)$. Thus $e$ is the normal $*$-representation of $\mathscr{A}(A, G)$ which uniquely extends $\left(\pi^{\prime}, L^{\prime}\right)$ and $e \otimes e$ is the induction on $\mathscr{A}(A, G) \otimes \mathscr{A}(A, G)$ with respect to the projection $E \otimes E$. Since $(\pi, L)$ and $\left(\pi^{\prime}, L^{\prime}\right)$ are quasi-equivalent, $\mathscr{A}(A, G)_{E}=\mathscr{A}\left(\pi^{\prime}, L^{\prime}\right)$ is also a Hopf von Neumann algebra and $e$ is a Hopf von Neumann algebra morphism of $\mathscr{A}(A, G)$ onto $\mathscr{A}(A, G)_{E}$. Note that if $T$ is an intrinsic element of $\mathscr{A}(A, G)$, then $e(T)=T_{E}$ is an intrinsic element of $\mathscr{A}(A, G)_{E}$.

Theorem 5.2. Let $(A, G)$ be a covariant system with enveloping Hopf von Neumann algebra $(\mathscr{A}(A, G), d)$. Then the canonical embedding of $G$ into $\mathscr{A}(A, G)$ is an isomorphism and $\sigma$-strong homeomorphism of $G$ onto the intrinsic group of $\mathscr{A}(A, G)$.

Proof. Earlier results establish that the canonical embedding of $G$ into $\mathscr{A}(A, G)$ is an isomorphism and $\sigma$-strong homeomorphism into the 
intrinsic group of $\mathscr{A}(A, G)$. It thus suffices to establish that the image is equal to the intrinsic group of $\mathscr{A}(A, G)$.

Let $T$ be an intrinsic element of $\mathscr{A}(A, G)$. Thus $e(T)=T_{E}$ is an intrinsic element of $\mathscr{A}(A, G)_{E}=\mathscr{A}\left(\pi^{\prime}, L^{\prime}\right)$. By Theorem 4.3, we have that $T_{E}$ is necessarily of the form $s_{E}$, for some $s$ in $G$. Thus $s^{-1} T$ is an intrinsic element of $\mathscr{A}(A, G)$ such that $\left(s^{-1} T\right)_{E}=S_{E}^{-1} T_{E}$ is the identity operator $\mathscr{A}(A, G)_{E}$.

Since $(\pi, L)$ is quasi-equivalent to $(\pi, L) Q(v, V)$ (cf. Theorem 4.2) we have $\left(\pi^{\prime}, L^{\prime}\right)$ is quasi-equivalent to $\left(\pi^{\prime}, L^{\prime}\right) Q(v, V)$. It therefore follows that their unique extensions to normal *-representations of $\mathscr{A}(A, G)$ are also quasi-equivalent. By the previous lemma,

$$
e \text { is quasi-equivalent to }(e \otimes i) \circ d \text {. }
$$

But note that $e$ maps $s^{-1} T$ into the identity operator $I$ on $\mathscr{H}\left(\pi^{\prime}, L^{\prime}\right)$. Thus $(e \otimes i) d\left(s^{-1} T\right)=e\left(s^{-1} T\right) \otimes S^{-1} T=I \otimes s^{-1} T$ must also be the identity operator on $\mathscr{H}\left(\pi^{\prime}, L^{\prime}\right) \otimes \mathscr{H}(v, V)$. Hence $s^{-1} T$ must be the identity operator on $\mathscr{H}(v, V)$. Thus $T=s$.

Corollary 5.3. Let $(A, G)$ be a covariant system and let $(\pi, L)$ be a left $\pi_{0}$-regular representation of $(A, G)$. Let $\pi^{\prime}$ be the corresponding normal *-representation of $\mathscr{A}(A, G)$. Then $\pi^{\prime}$ is a Hopf von Neumann algebra morphism of $(\mathscr{A}(A, G), d)$ onto $(\mathscr{A}(\pi, L), r)$ which maps the intrinsic group of $(\mathscr{A}(A, G), d)$ injectively onto the intrinsic group of $(\mathscr{A}(\pi, L), r)$.

Proof. This is precisely what we have just proved for the induction $\mathscr{A}(A, G) \rightarrow \mathscr{A}(A, G)_{E}$, and we have simply formulated it for the quasiequivalent normal $*$-representation $\pi^{\prime}$.

\section{§ 6. The Classical Formulation of Duality for the Automorphism Group of a Covariant System}

We wish to characterize the automorphism group $G$ of a covariant system $(A, G)$ as the set of options defined on the space of $\mathscr{R}(A, G)$ of covariant representations of $(A, G)$ which satisfies some axiom concerning the tensor product structure in $\mathscr{R}(A, G)$. In Section 2 we defined the concepts left and right tensor products, symmetric tensor products and left tensor squares. We shall see that any one of these concepts will suffice to give the needed structure to $\mathscr{R}(A, G)$.

Definition 6.1. An option $S$ defined on $\mathscr{R}(A, G)$ will be said to preserve left tensor products, if, whenever $\left(\pi_{1}, U_{1}\right)$ and $\left(\pi_{2}, U_{2}\right)$ are in $\mathscr{R}(A, G)$ and $u$ is a linear isometry of $\mathscr{H}\left(\pi_{1}, U_{1}\right) \otimes \mathscr{H}\left(\pi_{2}, U_{2}\right)$ onto a closed subspace 
of $\mathscr{H}_{0}$, such that

$$
u\left(\left(\pi_{1}, U_{1}\right) Q\left(\pi_{2}, U_{2}\right)\right) u^{-1} \in \mathscr{R}(A, G),
$$

then

$$
\begin{aligned}
& S\left(u\left(\left(\pi_{1}, U_{1}\right) \otimes\left(\pi_{2}, U_{2}\right)\right) u^{-1}\right) \\
& \quad=u\left(S\left(\pi_{1}, U_{1}\right) \otimes S\left(\pi_{2}, U_{2}\right)\right) u^{-1} .
\end{aligned}
$$

In a completely similar manner, we may define the notion: " $S$ preserves right tensor products", " $S$ preserves symmetric tensor products" and " $S$ preserves left tensor squares". We may also define "S preserves the left tensor square of the proper covariant representation $(\pi, U)$ " even when $(\pi, U)$ is not an element of $\mathscr{R}(A, G)$. Indeed $S$ has a canonical extension $S^{\prime}$ to the representations $(\pi, U)$ and $(\pi, U) Q(\pi, U)$ by requiring that $S^{\prime}$ preserve unitary equivalence and the direct sum of representations (cf. axioms iii) and iv) in the definition of a option, Definition 3.2 of [3]) and noting that $(\pi, U)$ and $(\pi, U) Q(\pi, U)$ are both direct sums of proper cyclic covariant representations (cf. Proposition 2.7 of [3]), each of which is unitary equivalent to an element of $\mathscr{R}(A, G)$. Then we say $S$ preserve the left tensor square of the proper covariant representation $(\pi, U)$ if

$$
S^{\prime}((\pi, U) Q(\pi, U))=S^{\prime}(\pi, U) \otimes S^{\prime}(\pi, U) .
$$

Theorem 6.2. Let $S$ be an invertible element of $\mathscr{A}(A, G)$, i.e., an invertible option defined on $\mathscr{R}(A, G)$. Then the following conditions are equivalent.

(i) $S=\hat{s}$ for some s in $G$, where $\hat{s}(\pi, U)=U(s)$ for all $(\pi, U)$ in $\mathscr{R}(A, G)$.

(ii) $S$ preserves the left-tensor square of a left- $\pi_{0}$-regular covariant representation of $(A, G)$.

(iii) $S$ preserves left tensor squares.

(iv) $S$ preserves left tensor products.

(v) S preserves right tensor products.

(vi) S preserves symmetric tensor products.

Proof. We first show that (ii) implies (i). Let $\pi^{\prime}$ denote the normal *-representation of $\mathscr{A}(A, G)$ corresponding to the left $\pi_{0}$-regular representation $(\pi, L)$. By definition of this extension $\pi^{\prime}(S)=S^{\prime}(\pi, L)$. Then (cf. Lemma 5.1) $\left(\pi^{\prime} \otimes \pi^{\prime}\right) \circ d$ is the extension of $(\pi, L) Q(\pi, L)$ to $\mathscr{A}(A, G)$ where $d$ is the comultiplication of $\mathscr{A}(A, G)$. Hence

$$
\begin{aligned}
{\left[\left(\pi^{\prime} \otimes \pi^{\prime}\right) \circ d\right](S) } & =S^{\prime}((\pi, L) \otimes(\pi, L)) \\
& =S^{\prime}(\pi, L) \otimes S^{\prime}(\pi, L) \\
& =\pi^{\prime}(S) \otimes \pi^{\prime}(S) .
\end{aligned}
$$


Since $\pi^{\prime}$ is a Hopf von Neumann algebra morphism of $(\mathscr{A}(A, G), d)$ onto $(\mathscr{A}(\pi, L), r)$ (cf. Corollary 5.3) we have

$$
\pi^{\prime} \otimes \pi^{\prime} \circ d=r \circ \pi^{\prime} .
$$

Hence

$$
r\left(\pi^{\prime}(S)\right)=\pi^{\prime}(S) \otimes \pi^{\prime}(S)
$$

Thus $\pi^{\prime}(S)$ is an intrinsic element of $\mathscr{A}(\pi, L)$. However (Corollary 5.3) $\pi^{\prime}$ maps the intrinsic group of $(\mathscr{A}(A, G), d)$ isomorphically onto the intrinsic group of $(\mathscr{A}(\pi, L), r)$. Thus $S$ is an intrinsic element of $\mathscr{A}(A, G)$ and hence (Theorem 5.2) $S$ is of the form $\hat{s}$, for some $s$ in $G$.

The equivalence of conditions iv) and $v$ ) follows from the fact that, for any pair $\left(\pi_{1}, U_{1}\right)$ and $\left(\pi_{2}, U_{2}\right)$ of covariant representations in $\mathscr{R}(A, G)$, $\left(\pi_{1}, U_{1}\right) Q\left(\pi_{2}, U_{2}\right)$ is unitary equivalent to $\left(\pi_{2}, U_{2}\right) Q\left(\pi_{1}, U_{1}\right)$ where the linear isometry is the usual mapping of $\mathscr{H}\left(\pi_{1}\right) \otimes \mathscr{H}\left(\pi_{2}\right)$ onto $\mathscr{H}\left(\pi_{2}\right) \otimes \mathscr{H}\left(\pi_{1}\right)$ and the fact that any option preserves unitary equivalence (cf. axiom iv) of Definition 3.2 of [3]). Hence we clearly havei) $\Rightarrow v i) \Rightarrow v) \Rightarrow$ iv) $\Rightarrow$ iii) $\Rightarrow$ ii).

Remark 6.3. In the duality Theorems 4.3, 5.2 and 6.2, the requirement that the intrinsic elements be invertible may be weakened to the requirement that they be non-zero. We have used the invertible condition because we like Proposition 3.5 and Proposition 3.5 is not true without this invertibility assumption. Indeed consider a von Neumann algebra $\mathscr{A}$ with a non-trivial projection $E$. The general element is $\lambda I+\mu E$, with $\lambda, \mu$ in $\mathbb{C}$. Define $d(\lambda I+\mu E)=\lambda I \otimes I+\mu E \otimes E$. Then $(\mathscr{A}, d)$ is a Hopf von Neumann algebra and $d(E)=E \otimes E$, where $E$ is non-zero and nonunitary. However Proposition 3.5 was not used in the proofs of the duality theorems. Indeed all of these theorems are proved by reducing to the case of the Hopf von Neumann algebra generated by the left regular representation of the automorphism group $G$. Here the Tatsuuma duality theory is applicable, and in this case one need only assume that the intrinsic elements are non-zero rather than invertible (cf. [6]).

\section{References}

1. Doplicher, S., Kastler, D., Robinson, D.: Covariance algebras in field theory and statistical mechanics. Commun. Math. Phys. 3, 1-28 (1966).

2. Ernest, J.: Hopf von Neumann algebras, Proceedings of the Functional Analysis Conference at Irvine, 195-215. Washington, D. C.: Thompson Book Comp. 1967.

3. - The enveloping von Neumann algebra of a covariant system. Commun. Math. Phys. 17, 61-74 (1970). 
4. Takesaki, M.: A duality in the representation theory of $C^{*}$-algebras. Ann. Math. 85, 370-382 (1967).

5. Tannaka, T.: Über den Dualitätssatz der nichtkommutativen topologischen Gruppen. Tôhoku Math. J. 53, 1-12 (1938).

6. Tatsumma, N.: A duality theorem for locally compact groups. J. Math. Kyoto Unıv. 6, 187-293 (1966-67).

John Ernest

Mathematics Department

University of California

Santa Barbara, California, 93106, USA 